\title{
COVID-19 community assessment hubs in Ireland-the experience of clinicians
}

\author{
Gerard Bury ${ }^{1}$ (D) $\cdot$ Susan Smith ${ }^{2} \cdot$ Maureen Kelly ${ }^{3} \cdot$ Colin Bradley $^{4} \cdot$ William Howard $^{5} \cdot$ Mairead Egan $^{1}$
}

Received: 7 August 2020 / Accepted: 22 September 2020 / Published online: 26 September 2020

(C) Royal Academy of Medicine in Ireland 2020

\begin{abstract}
Background COVID-19 required rapid innovation in health systems, in the context of an infection which placed healthcare professionals at high risk; general practice has been a key component of that innovative response. In Ireland, GPs were asked to work in a network of community assessment hubs. A focused training programme in infection control procedures/clinical use of personal protective equipment (PPE) was rapidly developed in advance. University departments of general practice were asked to develop and deliver that training.

Aim The aim of this article is to describe infection control procedure training in Ireland, the uptake by GPs and the initial experience of GPs working in this unusual environment.

Design and setting Two anonymous cross-sectional online surveys are sent to participants in training courses.

Method Survey 1 followed completion of training; survey 2 followed establishment of the hubs.

Results Six hundred seventy-five participants (including 439 GPs, 156 GP registrars) took part in the training. Two hundred thirty-nine $(50.3 \%$ ) out of four hundred seventy-five responded to Survey 1 - over $95 \%$ reported an increase in confidence in the use of PPE. Two hundred ten (44.2\%) out of four hundred seventy-five participants responded to Survey 2; 195 had completed hub shifts. Younger, female GPs predominated. Very high levels of infection control procedures were reported. Participants commented positively on teamworking, environment and systems. However, 'real-time' ambulance service data suggest the peak of the surge may have passed by the time the hubs were established.

Conclusion Academic departments, GPs and the Irish health system collaborated effectively to respond to the need for community assessment of COVID-19 patients.
\end{abstract}

Keywords COVID-19 $\cdot$ Infection control $\cdot$ Training

\section{Introduction}

By July 2020, Ireland had 25,500 confirmed COVID-19 cases and 1740 deaths related to the disease [1]. Around $60 \%$ of all deaths have occurred in residential care facilities and around

Gerard Bury

gerard.bury@ucd.ie

1 Academic General Practice, School of Medicine, UCD, Dublin, Ireland

2 Department of Family Medicine \& Primary Care, RCSI, Dublin, Ireland

3 Discipline of General Practice, NUIG, Galway, Ireland

4 Department of General Practice, UCC, Cork, Ireland

5 HSE National Ambulance Service, Dublin, Ireland
$30 \%$ of cases have been in healthcare workers; more than $50 \%$ of all cases have occurred in the greater Dublin area/eastern counties [2]. Given a population of $4.9 \mathrm{~m}$, these figures indicate high incidence and fatality rates. The importance of clinical, procedural, organisational and even ethical frameworks to minimise transmission of SARS-CoV2 among patients and healthcare staff is therefore very clear.

In March 2020, the HSE announced the establishment of around 50 'COVID-19 community assessment hubs' in which confirmed or presumptive cases of COVID-19 would be assessed by GPs, public health nurses (PHNs) and other clinical members of primary care teams, following referral by the patient's own doctor [3]. The HSE system closely reflects that established around the same time in the UK $[4,5]$.

On April 3, a HSE request was made to the university departments of general practice to support the training of those clinicians in their roles within the hubs. A half-day training 
course - 'Clinical PPE training' — was developed by the departments, with significant input from the National Ambulance Service (NAS) staff with experience and expertise in the use of personal protective equipment (PPE) from the already established COVID-19 testing sites.

GPs were invited to volunteer to work in hubs while GP registrars and primary care staff were directed by their employer, the HSE, to work in specified units. Those aged over 60 , with pre-existing health conditions or who were pregnant were advised not to take part. Hubs began to accept referrals during the week of 5 April and continue to operate in certain parts of Ireland, although now on a much-reduced basis.

This article reports on two follow-up surveys of the clinicians who undertook this training - the first was a simple demographic/feedback exercise for those who had participated in training and the second explored experience of working in the community assessment hubs. The purpose of both was to examine the experience of clinicians with a focus on improving their safety while working in this environment. The timing of hub availability - and therefore, potential exposure of clinicians to risk - is also considered by comparing Department of Health national data on daily case occurrence with 'real-time' COVID-19-related emergency calls to Dublin Fire Brigade Ambulance Service.

\section{Clinical PPE training course}

The principles underpinning immediate care training courses provided the framework, delivery by peers, significant practical content, clear links between skills training and underpinning clinical purpose and supervised small-group skills training; satisfactory completion required full attendance and completion of all tasks, without formal assessment [6].

A course curriculum was developed using these principles, with content and format agreed on a consensus basis - while use of PPE was central, undertaking simulated clinical work in a series of skills stations while rigorously observing infection control procedures making up the bulk of the course. The HSE nominated most candidates and provided funding and PPE supplies for each candidate and the universities provided access to their facilities. Candidates were asked to watch the standard HSE PPE training videos before attendance [7].

Courses were delivered in Dublin, Galway and Cork/Kerry. The settings were mainly large university sports halls which allowed for social distancing and candidates wore surgical masks and used hand gel throughout. Brief introductory and concluding sessions provided updates on SARSCoV2, epidemiology, aerosol generating procedures, operational principles for the hubs and demonstrations of 'donning' and 'doffing' PPE by experts. Due to evolving PPE supplies and specifications, the emphasis was on the principles of PPE use and on the rigorous use of demarcated 'clean' and 'dirty' areas to be established in the hubs.

Two hours of the course consisted of small groups circulating through taught simulated clinical scenarios while observing strict PPE and 'clean/dirty area' principles; cases included a 'worried well' patient, a COVID-19 patient with community-acquired pneumonia and a COVID-19 patient who developed cardiac chest pain.

The HSE logistics unit expeditiously provided PPE for each session; training focused on the use of gown/mask/ gloves but all candidates were also introduced to 'hazmat-type suits'/goggles/visors/FFP2/3 masks and particularly the challenges of safe doffing. All specifications of PPE were supplied to hubs at various times.

The HSE indicated that PHNs and other primary care staff would receive training within their own hubs-however, when requests were made by these colleagues to attend clinical PPE training, they were facilitated when possible.

\section{Methods}

Both surveys were carried out anonymously using 'Google surveys'. Because courses delivered in Cork/Kerry used a different method of contact, no email addresses were available and this group is not included in the study; no valid email addresses could be located for 18 other individuals and 41 messages were to non-responding addresses or to individuals who were not eligible to work in hubs, giving a denominator of 475 .

Survey 1 gathered demographic and satisfaction data while survey 2 explored the working environment and procedures and perceived exposure to risk; both surveys offered a free text comments section, which was analysed thematically. No attempt was made to examine workload, clinical content or outcomes of care. Data on activity within hubs has not yet been published by the HSE.

Exemption from full ethical approval was provided by the UCD Human Research Ethics Committee.

The study also reports data from Dublin Fire Brigade Ambulance Service (which provides most emergency ambulance services in the greater Dublin area) on COVID-19related calls from March to June 2020. The data provides a real-time daily context for the establishment of hub services [8].

\section{Results}

Eighteen courses were provided to 675 participants (439 GPs, 156 GP registrars, 69 PHNs and 11 primary care staff) from April 6 to April 23 by staff of the university departments and NAS volunteers at sites around the country. Training sites 
included Dublin (461 participants), Galway (72 participants) and Cork/Kerry (142 participants).

Valid email addresses were available for 475 course participants.

\section{Survey 1}

Two hundred thirty-nine (50.3\%) out of four hundred seventyfive participants responded to survey 1 (feedback on training) - this included $207 \mathrm{GPs} / \mathrm{GP}$ registrars. Table 1 summarises demographic characteristics of respondents. Most participants were female $(61.5 \%)$ and $41 \%$ were aged less than 46 years old.

Overall, 228 (95.4\%) reported that they had increased confidence in using PPE as a result of the training, $10(4.2 \%)$ reported somewhat increased confidence and one individual reported no change. Respondents were invited to make suggestions or comments on the training course and 192 (80.7\%) choose to do so - the vast majority of comments were positive and indicated the course met key needs:

'Initially i didn't think 4 hours was necessary to learn to don PPE but afterwards I didn't feel there was anything that I would have wanted cut from the program. Great job.'

'It was very well run. Very competent delivery by facilitators. We are all new to this \& the familiarising with the PPE removed my personal fear of using it. There will always be slight variations but the basic fundamental safe use was very well explained \& delivered.'

Suggestions included more operational information, greater consistency in types of PPE used and increasing or decreasing the time spent on practical skills.

Table 1 Respondents (training survey) by age, gender and profession ( $n$ =238)

\begin{tabular}{llllll}
\hline & GP & GP registrar & PHN & Other HCP & Total \\
\hline Male & & & & & 92 \\
$25-45$ & 33 & 0 & 0 & 1 & \\
$46-60$ & 36 & 17 & 0 & 0 & \\
$>60$ & 4 & 0 & 0 & 1 & 147 \\
Female & & & & & \\
$25-45$ & 47 & 0 & 7 & 10 & \\
$46-60$ & 40 & 31 & 5 & 5 & 239 \\
$>60$ & 0 & 0 & 0 & 2 & \\
Total & 159 & 48 & 12 & 18 & \\
\hline
\end{tabular}

\section{Survey 2}

Two hundred ten $(44.2 \%)$ out of four hundred seventy-five participants responded to survey 2 , of whom 15 had not completed any shifts within the hubs. The 195 clinicians who had completed shifts provide the denominator for reported experience - this included $170 \mathrm{GPs} / \mathrm{GP}$ registrars. Although all provinces were represented, $72 \%$ of all respondents worked in hubs in the east of the country (Leinster).

Table 2 summarises demographics and reported shift patterns and indicates that $126(64.6 \%)$ clinicians were female and $122(62.6 \%)$ were in the 25-45 age group. Most shifts were of $6 \mathrm{~h}$ or $12 \mathrm{~h}$ duration. Of 119 GPs, $34(28.6 \%)$ had completed more than five shifts. Of 51 GP registrars, 34 (65.4\%) had completed more than five shifts.

Supplies of PPE were said to be adequate by 194 (99.9\%) respondents.

In terms of compliance with PPE procedures, 181 (92.8\%) said compliance was very good and $14(7.2 \%)$ said compliance was adequate.

During their shifts, 23 (1.2\%) clinicians reported that aerosol generating procedures were carried out, with one respondent reporting more than three such interventions; no clinical information was gathered on the nature of those procedures.

Overall, 163 (83.6\%) clinicians reported that IT systems within the hubs were adequate or very good but $32(16.4 \%)$ described these systems as 'poor'. Many of the comments from respondents related to experience with the IT systems:

'I found the IT software difficult to use. Not at all intuitive. With a once weekly shift it felt like you had to learn it all over again each time.'

'IT system very cumbersome/not user friendly/apart from that the experience in the Hub has been excellent.'

The large majority of respondents felt that referral systems to the hubs and reporting systems back to referring GPs worked well; just 14 (7.2\%) clinicians felt that referral systems to the hubs were poor and $34(17.4 \%)$ clinicians felt that reporting systems back to the referring GP were poor. In addition, $20(10.3 \%)$ felt that ease of referral to support services was poor. Where deficits were noted, suggestions were offered for change. Overall, dissatisfaction with IT and administration systems focused on ease of use compared with mainstream GP electronic platforms, rather than on any identified deficits in the content. The hubs system used a 'Swiftqueue' regional booking system which appeared to work very efficiently while most comments related to the patient electronic record system are used in consultations.

Ninety $(46.2 \%)$ of respondents chose to make additional comments about their experiences. Key themes included: 
Table 2 Respondents (hub survey) by age, gender and profession $(n=195)$

\begin{tabular}{lrrrrrrrrr}
\hline & GP & \multicolumn{2}{c}{ GP registrar } & PHN & & Other HCP & Total \\
\hline Shifts & $1-5$ & $>5$ & $1-5$ & $>5$ & $1-5$ & $>5$ & $1-5$ & $>5$ & \\
Male & & & & & & & & & $69(40 \times 25-45)$ \\
$25-45$ & 16 & 6 & 7 & 11 & 0 & 0 & 0 & 0 & \\
$46-60$ & 15 & 11 & 0 & 0 & 0 & 0 & 0 & 1 & \\
$>60$ & 2 & 0 & 0 & 0 & 0 & 0 & 0 & 0 & \\
Female & & & & & & & & & $126(82 \times 25-45)$ \\
$25-45$ & 28 & 8 & 11 & 21 & 1 & 6 & 0 & 7 & \\
$46-60$ & 24 & 9 & 1 & 0 & 1 & 6 & 0 & 3 & \\
$>60$ & 0 & 0 & 0 & 0 & 0 & 0 & 0 & 0 & \\
Totals & 85 & 34 & 19 & 32 & 2 & 12 & 0 & 11 & \\
& 119 & & 51 & & & 14 & & 11 & \\
\hline
\end{tabular}

i. A very positive experience in the hubs and praise for the efficient and rapid establishment of the system

Despite the fact that the numbers attending the assessment Hubs were not as expected, it was an incredible achievement by all involved in getting the service up and running so efficiently. Day to day the Hub ran really well and all staff were a pleasure to work with.'

'Very supportive and safe environment. Excellent and motivated staff.'

'Good organisation, clear communication between the team. It was a lovely team to be part of.'

'Very positive rewarding experience personally \& professionally.'

'Brilliantly organised.'

'A good model and really important in supporting the efforts to keep general practices COVID-19 free and let other work take place etc.'

'The experience of working closely with PHNs has been a pleasure and will leave a positive legacy.'

'Overall thoroughly enjoyed being part of this experience. Much learned. Great connections made. Thanks for the training and confidence.'

ii. Limited and falling workload

'While a good initiative initially I feel that the resources allocated to the Hub outweigh the benefits. A large number of staff for few patients with knock on effects on public health nursing particularly.'
'The Hubs were/are very quiet. Max 3 patients in 4 hour shift, min 0 and mode 0 .'

'Hub quiet. Better be looking at it and not needing it than needing it and looking for it.'

'I've worked four shifts and in total only one patient was referred and seen during one of those shifts.'

iii. Potential for re-use of the model

'Currently I feel they are no longer needed and I strongly feel that the HSE should step them down with a view to opening them up again should they be needed.'

'Is there a need for HUBs at this time? Perhaps mothballing them till the need arises as numbers referred are very low and mostly don't need specifically Hub expertise. We know it is well set up and functioning, perhaps to consider holding them till the need arises and literally have them up and running within $24 \mathrm{hrs}$ as we know how it works.

'Well set up, may be a greater need for them in the autumn winter for all respiratory presentations to ease pressure in GP surgeries.'

iv. Preparatory training helped

'Training was excellent.... thank you!'

'The preparation for this exercise starting with the truly excellent PPE training, has been most impressive and a real morale boost.' 
'I'll be happy to work in the Hubs when a further wave occurs. Thank you for the training.'

Figure 1 illustrates the number of COVID-19-related emergency ambulance calls identified by the DFB Ambulance Service in the greater Dublin area together with the national report of confirmed cases of COVID-19. National reporting of COVID-19 cases was not necessarily a 'real-time' event, as the Department of Health stressed that these figures were compiled from many sources over varying time periods.

Figure 1 shows that the peak of COVID-19-related emergency ambulance calls was approximately 2 weeks earlier (7 April) than the peak of reported COVID-19 cases (25 April) and then fell rapidly.

\section{Discussion}

Irish general practice has responded at many levels to the COVID-19 pandemic [9]. This study describes the high level of general practice support in bringing community assessment hubs into operation, at a time of very significant COVID-19related demand on general practice, amid great change in normal operational routines. More than 500 GPs and GP registrars came forward to complete relevant clinical training in order to work in hubs, during April 2020 - this represents around $15 \%$ of the general practice population. Almost all HSE regions required completion of this training programme by GPs and GP registrars, so it is likely that participants represent the large majority of GPs and GP registrars who eventually worked in hubs.

Data on hub activity is not yet available from the health service but the original 50 hubs appear to have been rapidly reduced in numbers and opening hours as workload was evaluated. No data exists on the total number of clinicians who worked in hubs but the 170 GPs and GP registrars who responded to this study are likely to be a significant proportion of the doctors who carried out shifts and may therefore provide useful insights into this novel clinical setting.

It is striking that young, female doctors contributed so heavily to the operation of the hubs, with two-thirds of GP registrars having completed more than five shifts when only one-third of GPs had done so. Ireland has around 3000 GPs and approximately 300-340 GP registrars working in general practice. Because GPs could volunteer their services and the HSE advised against the involvement of older doctors, practices may have made their own decisions about which members of staff would participate, with a resulting emphasis on younger GPs with no health problems coming forward. Many respondents expressed concerns at the fact that GP registrars were required to work within hubs and were scheduled at a high level of activity, whereas GP principals were invited to volunteer and self-selected their workload.

Attendance at clinical PPE training was very high and participants reported high levels of satisfaction with the training and with the preparation they received for work in the hubs. Clinicians working in the hubs generally reported good working conditions in terms of availability of PPE and use of appropriate procedures; also, aerosol generating procedures seem to have been very infrequent.

The challenges of general practice have been much highlighted internationally in recent years in terms of increasing workload, financial difficulties, limited recruitment and poor morale. It is noteworthy that the sentiments of respondents working in hubs were significantly different - praise for the health service was strongly expressed, clinicians were enthusiastic about participation in the service and where criticism was offered, it was focused and constructive. Perhaps counterintuitively, involvement in an innovative clinical service perceived to be very challenging but of real importance seems to have had a significant positive effect on the morale of GPs, at a time when general practice itself was under tremendous pressure. The potential benefits of such positivity in
Fig. 1 Daily confirmed cases versus daily 999 ambulance calls in Dublin (30 March to 10 June). (series 1, DFB COVID-19 calls; series 2, confirmed COVID-19 cases)

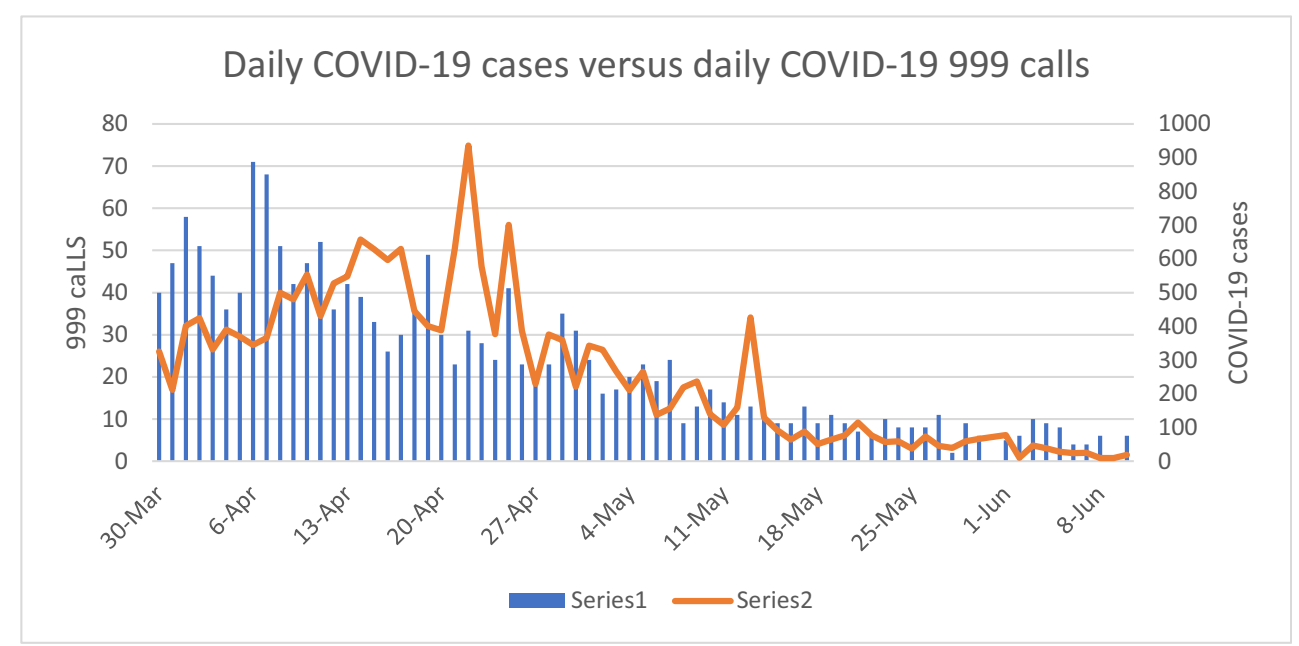


dealing with the many future COVID-19 challenges for general practice will be interesting to explore [10].

It is noteworthy that in times of unprecedented change, within a 3-week period, almost 600 GPs had completed standardised training at three national sites. Collaboration between academic departments, the health service and clinical experts within the National Ambulance Service allowed the urgent operational need for training to be identified and an effective solution developed and implemented within a period of days. The potential for collaborations like these to meet the needs of systems, staff and patients in the future should be acknowledged and developed further.

Many respondents reported low or falling levels of clinical activity and no respondent reported a high demand role, with reductions in hub availability being introduced later in April. The timing of the introduction of the hubs is an important potential learning opportunity for future COVID-19 surges. Emergency ambulance usage in the greater Dublin area peaked 2 weeks before the peak of reported positive cases, at which time emergency ambulance use had fallen by $50 \%$. Perhaps availability of hub services 2 or 3 weeks earlier might have better matched demand within the community. It seems likely that emergency ambulance use might in the future be a sensitive marker for morbidity in the community which might guide the need for re-introduction of hub services.

This study has significant limitations including limited responses to both surveys, potential self-selection by respondents with specific views or experience, the absence of any operational/utilisation data to provide context and limited potential depth using this survey strategy. However, the data represent a 'first look' at the contribution of GPs in Ireland to a major health crisis and provide insights into the experience and lessons learned by those doctors.

More formal evaluation of the utility of the hubs is required when national data on activity, costs and healthcare workerassociated morbidity become available and will guide system development. However, the contributions and experience of GPs and other primary care staff have been extremely positive and bode well for further iterations of the service.

Acknowledgements The authors thank the academic, general practice, nursing, DFB and NAS clinicians who came forward to develop and implement this programme and the administrative staff who made it happen.

Authors' contributions All authors have contributed to the design and preparation of the study.

Funding No funding support was provided for this study.

Data availability Data and materials can be made available by application.

\section{Compliance with ethical standards}

Conflict of interest No author has any conflict of interest.

\section{References}

1. Health Protection Surveillance Centre, Ireland. Coronavirus in Ireland. https://www.hpsc.ie/a-z/respiratory/coronavirus/ novelcoronavirus/. Accessed August 2020

2. DoH / NPHET

3. Health Service Executive, Ireland. https://www2.hse.ie/services/ coronavirus-community-assessment-Hubs/. Accessed August 2020

4. Primary Care Assessment Hubs, NHS Wales. http://www. powysthb.wales.nhs.uk/news/52429. Accessed July 2020

5. Community assessment centres. NHS Scotland. https://www. nhsggc.org.uk/your-health/health-issues/COVID-19-19coronavirus/for-nhsggc-staff/general-staff-guidance-resources/ community-assessment-centres-cacs/\#. Accessed July 2020

6. Barry T, Headon M, Glynn R et al (2018 May) Ten years of cardiac arrest resuscitation in Irish general practice. Resuscitation. 126:4348. https://doi.org/10.1016/j.resuscitation.2018.02.030

7. Health Protection Surveillance Centre, Ireland. Use of PPE. https:// www.hpsc.ie/a-z/respiratory/coronavirus/novelcoronavirus/ guidance/infectionpreventionandcontrolguidance/ videoresourcesforipc/. Accessed August 2020

8. Keely D (2020) Chief Fire Officer, DFB. (Personal communication)

9. COVID-19 and Irish general practice. ICGP. https://www.icgp.ie/ index.cfm?spKey=in_the _practice.clinical_hub.COVID-19_19 coronavirus. Accessed August 2020

10. Khan N, Jones D, Grice A et al (2020) A brave new world: the new normal for general practice after the COVID-19 pandemic. BJGP Open. https://doi.org/10.3399/bjgpopen20X101103

Publisher's note Springer Nature remains neutral with regard to jurisdictional claims in published maps and institutional affiliations. 